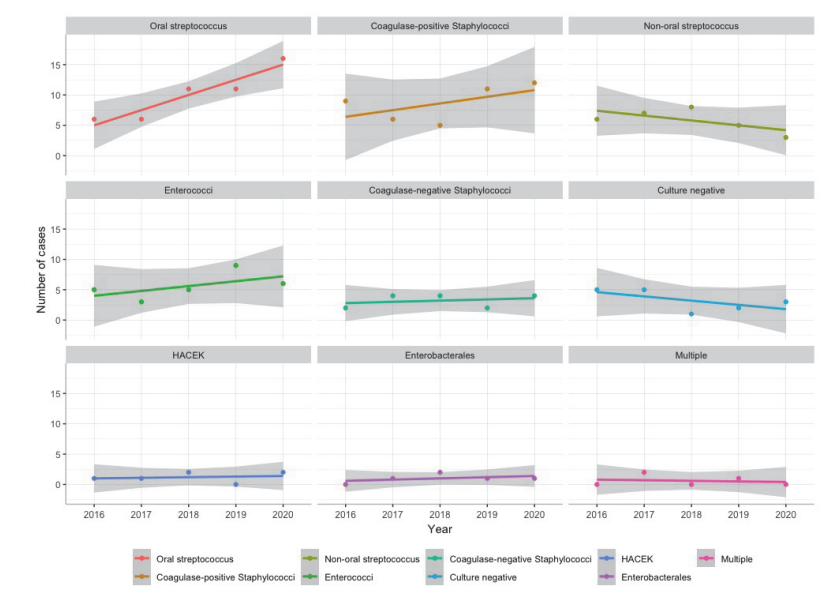

Abstract 11 Figure 1

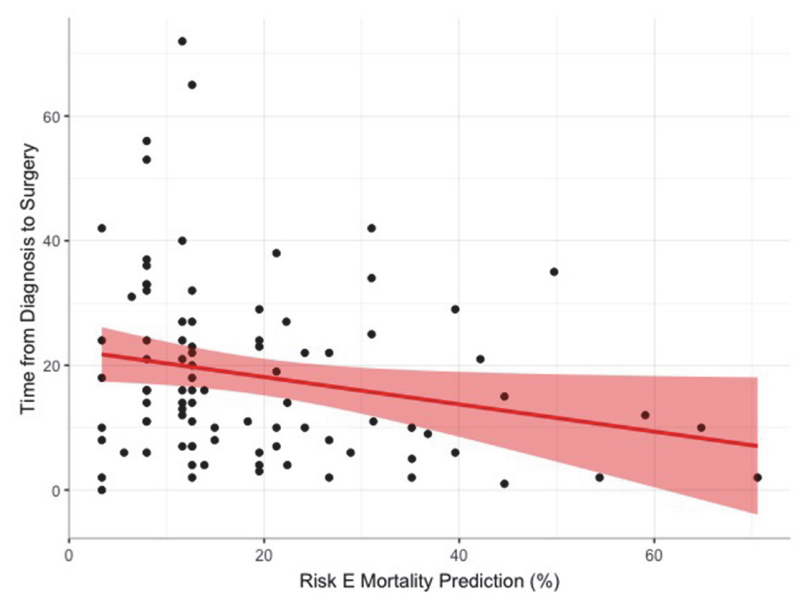

Abstract 11 Figure 2

management occurred in $46 \%$ of cases $(n=96)$. Transferred patients were more likely to undergo operative management (OR 3.3 , 95\% CI: 1.8, 5.8). Although men were more likely to undergo surgical management than women (OR 2.0, $95 \% \mathrm{CI}: 1.1,4.2)$, this is explained by women being six times more likely to be IVDUs (OR 6.1 CI: 2.3, 16.3) and have right-sided IE (OR 6.2 CI: 2.2, 17.4). When including only left-sided IE, the rate of surgery was not different between males and females $(p=0.1)$. There was no difference in in-hospital or 12-month mortality in patients admitted directly to SCC and those referred $(p=0.56)$ and there was no mortality difference between the sexes $(p=0.20)$. In-hospital mortality of surgically managed patients was predicted by the Risk-E Score and EuroSCORE II as 18 and 12 cases respectively. We observed 11 in-hospital deaths. This difference was not significant $(p=0.22)$. Transferred patients had a higher mean Risk-E Score $(\mathrm{p}<0.05)$ and a higher score was associated with a shorter time between diagnosis and surgery $(\mathrm{p}<0.05$, figure 2).

Conclusions There has been an increase in IE cases managed at the SCC over the last 5 years, with more transfers from other hospitals. This could be due to an increase in absolute IE numbers as well as better awareness of the need for early surgery and the involvement of an IE MDT. We report a previously unidentified male predominance in IE across all major organisms and a significant increase in oral streptococci infection; both require further exploration. In this population the Risk-E Score tended to overestimate in-hospital mortality. Larger multi-centre studies are required to explore these trends in sub-grouped populations.

Conflict of Interest None

\section{CLINICAL OUTCOMES UP TO 10 YEARS OF ASYMPTOMATIC INCIDENTAL AORTIC DISSECTIONS AND LARGE AORTIC ANEURYSMS DETECTED ON COMPUTER TOMOGRAPHY (CTA) PRIOR TO TRANSCATHETER AORTIC VALVE IMPLANTATION (TAVI)}

Joon Tan, Tsu Kuan Teoh, Joanna Fong, Asthika Amirthalingam, Kamran Baig. Nottingham University Hospitals NHS Trust, Nottingham, UK

\subsection{6/heartjnl-2021-BCS.12}

Introduction Computer Tomography Aortography (CTA) is performed routinely to aid planning of transcatheter aortic valve implantation (TAVI). Incidental findings are common, and may influence the decision to proceed with TAVI. The safety and long term outcomes of performing TAVI in patients with previously undiagnosed incidental CT findings of aortic dissections and large aortic aneurysms is currently unknown. We sought to establish the frequency of incidental aortic dissections and large aortic aneurysms prior to TAVI, and subsequent clinical outcomes of patients.

Methods This was a retrospective study of 628 sequential TAVI patients in a large, UK tertiary centre between January 2010 and September 2020. Patients were evaluated in 3 groups as per pre-TAVI CTA: incidental aortic dissections, aortic aneurysm $>4 \mathrm{~cm}$ and all others (control group). Endpoints were procedural success, peri-procedural major bleeding and/or vascular complications and/or CVA, length of hospital stay, 30-day and 1-year mortality.

Results $2.9 \%$ of patients $(n=18)$ had incidental aortic dissection, of which $66.7 \%(\mathrm{n}=12)$ were male, with a mean age of $86.7 \pm 4.4$. $3.8 \%$ of patients $(n=24)$ had aortic aneurysms $>4 \mathrm{~cm} .83 .3 \%(\mathrm{n}=20)$ of them were male and the mean age was $82.8 \pm 5.4$. $5.6 \% \quad(n=1)$ of the patients with incidental aortic dissection had severe left ventricular systolic dysfunction compared to $4.2 \%(n=1)$ of the patients in the aortic aneurysms group. Transfemoral approach was favoured in $77.8 \%$ of patients in the dissection group and $83.3 \%$ of patients in the aneurysm group versus $93.3 \%$ in the control group. A summary of procedural characteristics is summarised in table 1 . Vascular access complications, stroke, bleeding and length of hospital stay were comparable between all 3 groups (table 2). Patients with dissection and large aneurysm had a lower

Abstract 12 Table 1 Baseline patient and procedural characteristics $(n=628)$

\begin{tabular}{|l|r|r|r|}
\hline & $\begin{array}{r}\text { Incidental dissection } \\
(\mathrm{n}=18)\end{array}$ & \multicolumn{1}{|c|}{$\begin{array}{c}\text { Incidental aortic aneurysm } \\
(\mathrm{n}=24)\end{array}$} & $\begin{array}{c}\text { Other patients } \\
(\mathrm{n}=586)\end{array}$ \\
\hline Percutaneous transfemoral access & $10(55.6)$ & $20(83.3)$ & $497(84.8)$ \\
\hline Surgical transfemoral access & $4(22.2)$ & $0(0)$ & $50(8.5)$ \\
\hline Transapical access & $4(22.2)$ & $3(12.5)$ & $32(5.5)$ \\
\hline Transaartic access & $0(0)$ & $0(0)$ & $6(1)$ \\
\hline Axillary cutdown for access & $0(0)$ & $0(0)$ & $1(0.2)$ \\
\hline General anaesthesia & $9(50)$ & $7(29.2)$ & $132(22.5)$ \\
\hline TOE guidance & $9(50)$ & $7(29.2)$ & $128(21.8)$ \\
\hline Pre BAV & $17(89.5)$ & $17(70.8)$ & $408(69.6)$ \\
\hline Valve in valve TAVI & $1(5.6)$ & $2(8.3)$ & $32(5.5)$ \\
\hline Valve not deployed & $2(11.1)$ & $3(12.5)$ & $17(2.9)$ \\
\hline
\end{tabular}

Values are presented as mean \pm standard deviation or $N(\%)$. TOE $=$ Transoesophageal echocardiogram. $\mathrm{BAV}=$ Balloon aortic valvuloaltasty. $\mathrm{TAV}(\%)$. Transcatheter aortic valve implantation
Tran 
Abstract 12 Table 2 Clinical outcomes and mortality

\begin{tabular}{|l|r|r|r|}
\hline & $\begin{array}{c}\text { Incidental dissection } \\
(\mathrm{n}=18)\end{array}$ & $\begin{array}{c}\text { Incidental aortic aneurysm } \\
(\mathrm{n}=24)\end{array}$ & $\begin{array}{c}\text { Other patients } \\
(\mathrm{n}=586)\end{array}$ \\
\hline Success of valve deployment & $16(88.9)$ & $21(87.5)$ & $569(97.1)$ \\
\hline New Aortic dissection & $0(0)$ & $0(0)$ & $1(0.2)$ \\
\hline Vascular injury leading to death/limb amputation & $0(0)$ & $0(0)$ & $1(0.2)$ \\
\hline Unplanned endovascular/surgical intervention & $0(0)$ & $0(0)$ & $27(4.6)$ \\
\hline Stroke & $0(0)$ & $0(0)$ & $17(2.9)$ \\
\hline Major bleeding requiring transfusion & $1(5.6)$ & $5.7 \pm 6.6$ & $8.2 \pm 13.3$ \\
\hline Mean length of stay in hospital (days) & $9.7 \pm 13$ & $0(0)$ & $33(5.6)$ \\
\hline 30 day mortality & $4(22.2)$ & $6(25)$ & $81(13.8)$ \\
\hline 1 year mortality & $6(33.3)$ & & \\
\hline
\end{tabular}

Values are presented as mean \pm standard deviation or $\mathrm{N}(\%)$

\section{Kaplan-Meier anaylsis of 24 month MACE- free survival}

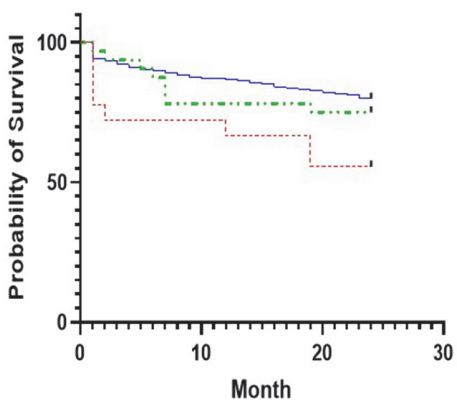

Abstract 12 Figure 1 Kaplan-Meier survival curve

success rate of valve implantation $(88.9 \%$ and $87.5 \%$ vs $97.1 \%, \mathrm{p}=0.01)$. 30-day mortality in the dissection group was higher than the other 2 groups $(21.1 \%$ vs $0 \%$ and $5.6 \%$, $\mathrm{p}=0.004)$. Log-rank analysis revealed a higher incidence of MACE in the dissection group over 24 months compared to the other two groups (figure 1).

Conclusion A transfemoral approach appears to be a safe choice in patients with incidental findings of aortic dissection or aortic aneurysms $>4 \mathrm{~cm}$. However, patients with stable previous aortic dissections have a significantly higher 30-day mortality and overall lower survival rate over 24 months. This important observation needs to be further investigated in a larger-scale, long-term follow up study, and may in future influence TAVI treatment planning.

Conflict of Interest None

\section{CLINICAL INFORMATICS TO DIRECT COMMUNITY ECHOCARDIOGRAPHY: AN ELECTRONIC HEALTHCARE RECORD PILOT}

${ }^{1}$ Kamatamu Amanda Mbonye, ${ }^{1}$ Alireza Yazdi, ${ }^{2}$ Shane Cashin, ${ }^{2}$ Nikhil Ahluwalia, ${ }^{2}$ Nabila Laskar, ${ }^{2}$ Sanjeev Bhattacharyya, ${ }^{2}$ Carl Hayward, ${ }^{2}$ Guy Lloyd, ${ }^{2}$ Abhishek Joshi. ${ }^{1}$ Royal London Hospital, Bart's NHS Trust; ${ }^{2}$ Bart's Heart Centre, London, UK

\subsection{6/heartjnl-2021-BCS.13}

Background Delayed diagnosis of valvular heart disease carries a poor prognosis, and early identification is desirable. We undertook a retrospective analysis of echocardiographic and electronic health care record data from the largest single cardiovascular service in the UK, to identify the burden of acute presentations with previously undiagnosed valvular heart disease and to determine the geographical and demographic distribution.

Methods and Results Automated text mining analysis was retrospectively applied to all echocardiographic examinations performed between 2015 and 2019 at Bart's Health NHS trust, identifying 2043 reports containing text or numerical data indicating severe valvular lesions. Demographic and clinical data was integrated with the echocardiographic dataset,

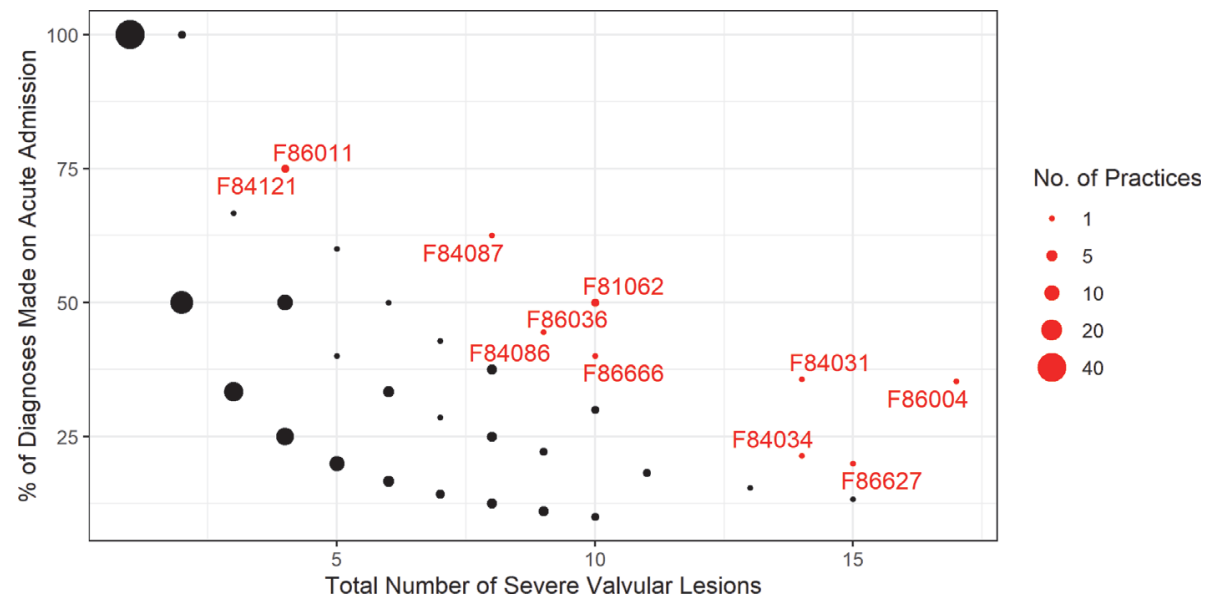

